\title{
Diacronie
}

Studi di Storia Contemporanea

$N^{\circ} 27,3$ | 2016

Stato, costituzione e democrazia

\section{Márcia Tiburi, Como conversar com um fascista. Reflexões sobre o cotidiano autoritário brasileiro}

\section{Karl Schurster}

Traduttore: Jacopo Bassi

\section{OpenEdition}

\section{Journals}

\section{Edizione digitale}

URL: http://journals.openedition.org/diacronie/4202

DOI: 10.4000/diacronie.4202

ISSN: 2038-0925

\section{Editore}

Association culturelle Diacronie

\section{Notizia bibliografica digitale}

Karl Schurster, « Márcia Tiburi, Como conversar com um fascista. Reflexões sobre o cotidiano autoritário brasileiro », Diacronie [Online], № 27, 3 | 2016, documento 18, Messo online il 29 septembre 2016, consultato il 24 septembre 2020. URL : http://journals.openedition.org/diacronie/4202 ; DOI : https:// doi.org/10.4000/diacronie.4202 


\section{RECENSIONE:}

\section{TIBURI, Márcia, Como conversar com um} fascista. Reflexões sobre o cotidiano autoritário brasileiro, Rio de Janeiro, Record, 2015, 194 pp.

a cura di Karl SCHURSTER *

traduzione di Jacopo BASSI

«Ciò che è in gioco è la riduzione dell'altro a un oggetto»1. Questa frase, tratta dall'ultimo libro della filosofa e artista gaúcha Márcia Angelita Tiburi forse è quella che meglio riassume tutto lo sforzo dell'autrice per far comprendere come agisca l'individuo fascista. Il suo intento principale è quello di analizzare, attraverso 67 brevi saggi, ciò che rimane del fascismo nella società attuale e quanto sia capace di produrre una soffocante quotidianità autoritaria. Niente di più opportuno nell'attuale congiuntura politica brasiliana.

L'8 marzo del 2016 l'Assemblea Legislativa dello Stato di Pernambuco, nel Nordeste brasiliano, ha cominciato a trattare un progetto di legge, presentato dal deputato Joel da Harpa appartenente al neonato Partido Republicano da Ordem Social (PROS), che dispone la proibizione dell'insegnamento di qualsiasi tematica relativa all'ideologia di genere in ambito educativo ${ }^{2}$. Questo progetto difende il divieto totale di discutere tematiche relative alla questione di genere cercando nella legislazione brasiliana un apparato atto a difendere un modello di famiglia che viene sistematicamente ridefinito attraverso un grande spazio di lotta e mobilitazione di gruppi e movimenti sociali. Il deputato fa ricorso nell'incipit del suo progetto all'aggettivo "difeso", derivante dal

\footnotetext{
${ }^{1}$ TIBURI, Márcia, Como conversar com um fascista. Reflexões sobre o cotidiano autoritário brasileiro, Rio de Janeiro, Record, 2015, p. 191.

2 Per approfondire, cfr., URL:

$<$ http://200.238.101.22/docreader/docreader.aspx?bib=20160308\&pasta=Mar\%C3\%A7o $\backslash$ Dia

\%2008 > [consultato il 26 maggio 2016].
} 
latino defensus, "proibito", per legiferare sul divieto dei docenti di trattare di questo argomento. È in questo spazio di discussione, su temi controversi, all'interno di una democrazia imperfetta, che si sono preservati gli spazi per quelle pratiche autoritarie che vengono inquadrate nelle analisi di Tiburi. Il suo intento è quello di promuovere un dibattito attraverso cui sia possibile stabilire un dialogo tra idee, proposte e persone che agiscono sotto l'egida del pensiero fascista utilizzando come principale strumento quello che è da tenere più caro trattando di fascismo: il dialogo. Per quale ragione è così importante per il deputato - appartenente a un milieu religioso e proveniente da un partito conservatore - mettere all'ordine del giorno dell'Assemblea Legislativa un progetto di legge che va contro i piani statali, municipali e nazionali in materia di istruzione? La sua proposta mira a evitare il dialogo e a normare ciò che l'altro può e deve essere, esattamente come una proposta fascista, o meglio, fascistizzante. In questo caso l'autrice ci fornisce una propria definizione di fascismo attraverso diversi esempi e riflessioni filosofiche, molte simili alle conclusioni a cui siamo giunti nella nostra tesi di dottorato ${ }^{3}$. Il fascismo per lei, come per noi, va molto oltre i limiti della politica o anche dell'economia, è all'interno di un campo filosofico nel quale il centro di gravità è qualcosa d'altro: nel caso dei fascismi, la negazione dell'altro. I testi risultano quindi un elemento di giunzione tra la filosofia di Theodor Adorno, il pensiero di Tzvetan Todorov e l'interpretazione della cultura di Homi Bhabha4.

Il testo da cui nasce il libro, Como conversar com um fascista, è una piccola, ma profonda, riflessione su come diverse pratiche sociali, quando non istituzionali, abbiano prodotto nel Brasile contemporaneo l'estinzione della politica. Questo annichilimento di quella che sarebbe per eccellenza l'arte del dialogo avrebbe generato il terreno fertile per la crescita dell'azione politica fascista e sarebbe presente nel nostro quotidiano attraverso ciò che la filosofia ha identificato come il «genocidio indigeno, il massacro razzista e classista contro i giovani neri e i poveri nelle periferie delle grandi città, la violenza domestica e l'assassinio delle donne, l'omofobia e la manipolazione dei bambini»5. Stando così le cose, il dubbio persistente risiede, qui, nella capacità della società di creare meccanismi capaci di impedire la ripetizione di fenomeni di autoritarismo e di odio attraverso una spiegazione rigorosa, adeguata e allo stesso tempo consistente, per $\mathrm{i}$ fenomeni dell'agire politico fascista in grado di negare l'esistenza dell'altro. Uno degli esempi più significativi di questo tentativo si ebbe nella

3 SCHURSTER, Karl, A história do tempo presente e a nova historiografia sobre o Nacional Socialismo, Tesi di Dottorato - Storia comparata, Universidade Federal do Rio de Janeiro, 2012. 4 Cfr. BHABHA, Homi K., I luoghi della cultura, Roma, Meltemi, 2001 [Ed. originale The location of culture, London, Routledge, 1994].

5 TIBURI, Márcia, op. cit., p. 29. 
stessa Germania, dove - malgrado l'enorme sforzo di denazificazione della società attuato subito dopo il 1945 e l'impatto del maggio 1968 - la società si mostrò incapace di offrire alle nuove generazioni strumenti critici per il superamento della seduzione del nazismo e dell'estremismo, consentendo l'emergere di un'ampia fascia di giovani affascinati dall'estremismo razzista e lo sviluppo dell'odio ${ }^{6}$. Lo stesso avviene con il nostro tempo presente. Trascorsi trent'anni dal processo di ridemocratizzazione del Brasile, ancora non siamo riusciti ad avanzare in forma sostanziale nella costruzione di una coscienza collettiva che privilegi la convivenza con le differenze. Viviamo ancora in un paese, come sostiene la filosofa, dove la differenza è qualcosa di insopportabile, dove le pratiche e i crimini dettati dall'odio passano come qualcosa di naturale o storicamente giustificabile. La professoressa Tiburi riafferma l'idea per cui le passioni sono sempre frutto di un apprendimento e si formano in noi attraverso l'esperienza. Il fascista, perciò - o colui che utilizza il fascismo come azione politica - è quell'individuo che ha vissuto e vive costanti esperienze di odio e diviene, con il passare del tempo, incapace di provare affetto per l'altro. Parafrasando l'autrice, questo individuo è stato ed è capace di introiettare l'«odio molto prima di riuscire a pensarlo»7 .

È chiaro, in considerazione delle tematiche affrontate nel libro di Tiburi - la crisi e il fallimento della politica nella quotidianità brasiliana, la democrazia e l'autoritarismo, la questione di genere, con un'analisi su ciò che ha chiamato la logica dello stupro, la questione dell'aborto, la paura e l'odio in televisione, i problemi etnici-razziali, specialmente per ciò che riguarda la questione indigena - che l'autoritarismo è molto più che presente nella vita brasiliana. È stato sistematicamente trasformato in una pratica giornaliera e banale, producendo quello che Félix Guattari ha chiamato i microfascismi ${ }^{8}$. Perché il fascismo sia una realtà, sostiene, bisogna che sia alimentato da pratiche quotidiane, da una routine che lo riproduca e che sia in grado di normalizzarlo. Questo non sarebbe quindi solamente un modo di riprodurre il fascismo come pratica e alternativa, ma, ancor più, di sterminio della democrazia, non quella che abbiamo oggi, di facciata, ma quella che desideriamo, a cui ambiamo. Ciò che risulta abbastanza evidente nel libro è che, fino ad ora, tutte le trasformazioni intraprese dalla società o dalle istituzioni sociali come la scuola non sono state sufficienti a formare una nuova gioventù critica e svincolata dai brutali atti di razzismo e di violenza, simbolica e

\footnotetext{
${ }^{6}$ Per approfondire si veda il documentario: Heil Hitler, Herr Lehrer! (LISKA, Peter, Heil Hitler, Herr Lehrer! Jugend unterm Hakenkreuz, 3sat, Germania, 2010, 50'). Il film è ricavato dal libro di Jürgen Kleindienst. Cfr. KLEINDIENST, Jürgen, Herr Lehrer: die Kindheit unter dem Hakenkreuz, 1933-1939, Frankfurt am Main, JKL, 1985.

7 TIBURI, Márcia, op. cit., p. 30.

8 Per approfondire l'argomento si veda: GUATTARI, Félix, Revolução molecular. Pulsações políticas do desejo, São Paulo, Brasiliense, 1981.
} 
fisica, contro l'altro. Nelle strade, negli stadi di calcio, nei bar e persino negli ambienti di lavoro si moltiplicano gli atti di razzismo e di esclusione, come sottolinea l'autrice del libro9. Riusciremo a superare, discutendone in modo critico, ciò che è già stato chiamato il fascino, der schöne Schein, di una cultura della violenza e del rifiuto dell'altro nel nostro quotidiano? Il libro risponde a questa domanda in modo sufficientemente obiettivo, affermando che solo attraverso il dialogo e il ritorno alla politica saremo in grado di rigettare l'agire fascista non considerandolo come un alternativa.

Il volume, inoltre, affronta, a partire da Nietzsche e dalla sua teoria dell'eterno ritorno, il cosiddetto peso del rancore, di quello che non può essere dimenticato, di ciò che ogni individuo e società sopporta per un lungo periodo. Bisogna comprendere in certi contesti quali siano i mezzi attraverso cui si produce il risentimento e come questi stessi siano in grado di creare uno spazio per la disseminazione dell'odio. Gli esempi attuali del riemergere dell'odio si sono moltiplicati negli ultimi anni, specialmente in Brasile: contro i neri, le donne, gli omosessuali, i migranti interni o coloro che sono immigrati per ragioni politiche o economiche. Persino nell'ambito degli sport di massa, in particolare nel calcio, la moltiplicazione degli atti di razzismo - non sempre affrontati con la necessaria severità dalle autorità responsabili - e spesso, troppo spesso, perpetrati da giovani, mostrano nel contesto della crisi economica globale, con un tasso di disoccupazione elevato e una frustrazione collettiva, un grande vuoto nel campo della rappresentanza politica e una mancanza di azioni da parte della società civile per porre fine all'incessante costruzione di quel che Peter Gay ha chiamato «l'altro conveniente» ${ }^{10}$.

I fallimenti e le omissioni del nostro processo educativo sono stati in grado di porre un freno all'emancipazione da questo atto e hanno posto le condizioni perché potesse prosperare e risorgere l'odio razziale, di classe, di gruppo, di genere e contro tutti coloro che vengano identificati intenzionalmente o meno, come un "altro" essenzialmente diverso. L'analisi di questi temi è il centro di gravità di gran parte dei capitoli del libro, attraverso vari esempi che disvelano la quotidianità della società brasiliana.

I diversi saggi che riflettono sulla questione femminile e su tematiche in cui la discussione è molto accesa, come lo stupro e la legalizzazione dell'aborto, risultano

\footnotetext{
9 Cfr. l'articolo su «El País»: ALTARES, Guillermo, «Cerca de 26\% dos judeus europeus dizem ter sofrido preconceito por causa de sua religião», in El País, 6 maggio 2014, URL: < http://brasil.elpais.com/brasil/2014/o6/o5/sociedad/1401978023_851631.html > [consultato il 22 maggio 2016]. Su un altro fronte, gli atti di razzismo contro i neri, i beurs, i pardos, persino se si tratta di celebri giocatori di calcio, si moltiplicano con estrema frequenza e, quando i loro perpetratori vengono identificati, ci sorprendiamo per la loro giovanissima età. ${ }^{10}$ GAY, Peter, O Cultivo do Ódio, São Paulo, Companhia das Letras, 1996.
} 
dunque fondamentali per avvalorare la difesa costante da parte dell'autrice del rispetto, della tolleranza, della diversità come elementi in grado di inibire la moltiplicazione di sintomi, atti e comportamenti permanenti di discriminazione e di odio, molto spesso legati ad un semplice e brutale disegno volto al ritorno ad un passato che è stato vissuto come un trauma. È in quest'ottica che il libro si trasforma in una lettura necessaria per comprendere il Brasile e le sue attuali sfaccettature. Attraverso l'interpretazione di Márcia Tiburi, è possibile capire che anche le democrazie consolidate dal punto di vista istituzionale, come quella brasiliana, corrono rischi costanti quando le pratiche fascistizzanti diventano una faccenda quotidiana. 


\section{* L'autore}

Karl Schurster è stato ricercatore post-doc e ha conseguito il dottorato in Storia presso l'UFRJ (Universidade Federal do Rio de Janeiro). Insegna come professore dell'Universidade de Pernambuco nel campo di studi della Storia del Tempo presente ed è membro permanente del corso di Laurea in Scienze della Formazione presso la stessa università. Attualmente sta realizzando un secondo stage postdottorale presso la Freie Universität Berlin sotto la direzione del professor Stefan Rinke. I suoi interessi sono rivolti allo studio della politica internazionale, con particolare interesse sui conflitti, in particolare le guerre mondiali e l'olocausto. È stato vincitore del $2^{\circ}$ posto al Premio Jabuti, assieme a Francisco Carlos Teixeira e Francisco Eduardo Almeida per il coordinamento dell'Atlântico: a história de um oceano, Rio de Janeiro, Editora Civilização Brasileira, 2013.

URL: < http://www.studistorici.com/progett/autori/\#Schurster >

\section{Per citare questo articolo:}

SCHURSTER, Karl, «Recensione: Recensione: TIBURI, Márcia, Como conversar com um fascista. Reflexões sobre o cotidiano autoritário brasileiro, Rio de Janeiro, Record, 2015, 194 pp.», Diacronie. Studi di Storia Contemporanea : Stato, costituzione e democrazia, 29/09/2016, URL:< http://www.studistorici.com/2016/09/29/schurster_numero_27/ >

Diacronie Studi di Storia Contemporanea $\approx$ www.diacronie.it

Risorsa digitale indipendente a carattere storiografico. Uscita trimestrale. redazione.diacronie@hotmail.it

Comitato di redazione: Jacopo Bassi - Luca Bufarale - Elisa Grandi - Antonio César Moreno Cantano - Deborah Paci - Fausto Pietrancosta - Alessandro Salvador - Matteo Tomasoni - Luca Zuccolo

Diritti: gli articoli di Diacronie. Studi di Storia Contemporanea sono pubblicati sotto licenza Creative Commons 3.0. Possono essere riprodotti e modificati a patto di indicare eventuali modifiche dei contenuti, di riconoscere la paternità dell'opera e di condividerla allo stesso modo. La citazione di estratti è comunque sempre autorizzata, nei limiti previsti dalla legge. 